\title{
Upper Abdomen
}

National Cancer Institute

\section{Source}

National Cancer Institute. Upper Abdomen. NCI Thesaurus. Code C139562.

The superior portion of the abdomen. 